\title{
Molecular Dynamics of a Water-Absorbent Nano-Scale Material Based on Chitosan
}

Carlos H. Borca ${ }^{1,2}$ and Carlos A. Arango ${ }^{2}$

${ }^{1}$ Departament of Chemistry, Purdue University, West Lafayette, Indiana, USA.

${ }^{2}$ Departamento de Ciencias Químicas, Universidad Icesi, Cali, Valle del Cauca, Colombia.

\section{Supporting information}

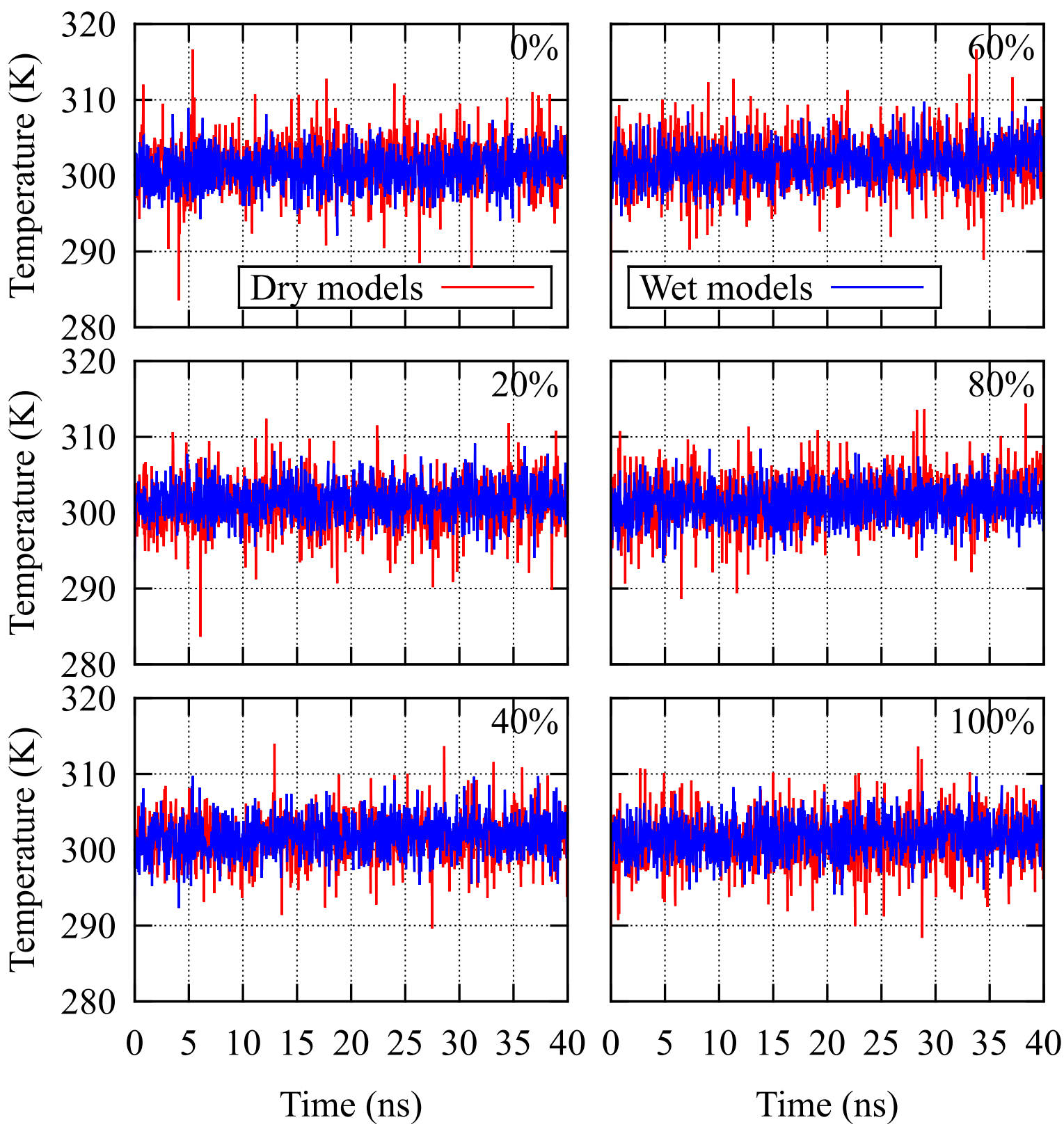

Figure S1: Time evolution of the temperature for the set of protonated models. Averages taken at intervals of $20 \mathrm{ps}$. Both the equilibration (0-20 ns) and production runs (20-40 $\mathrm{ns})$ are shown. 

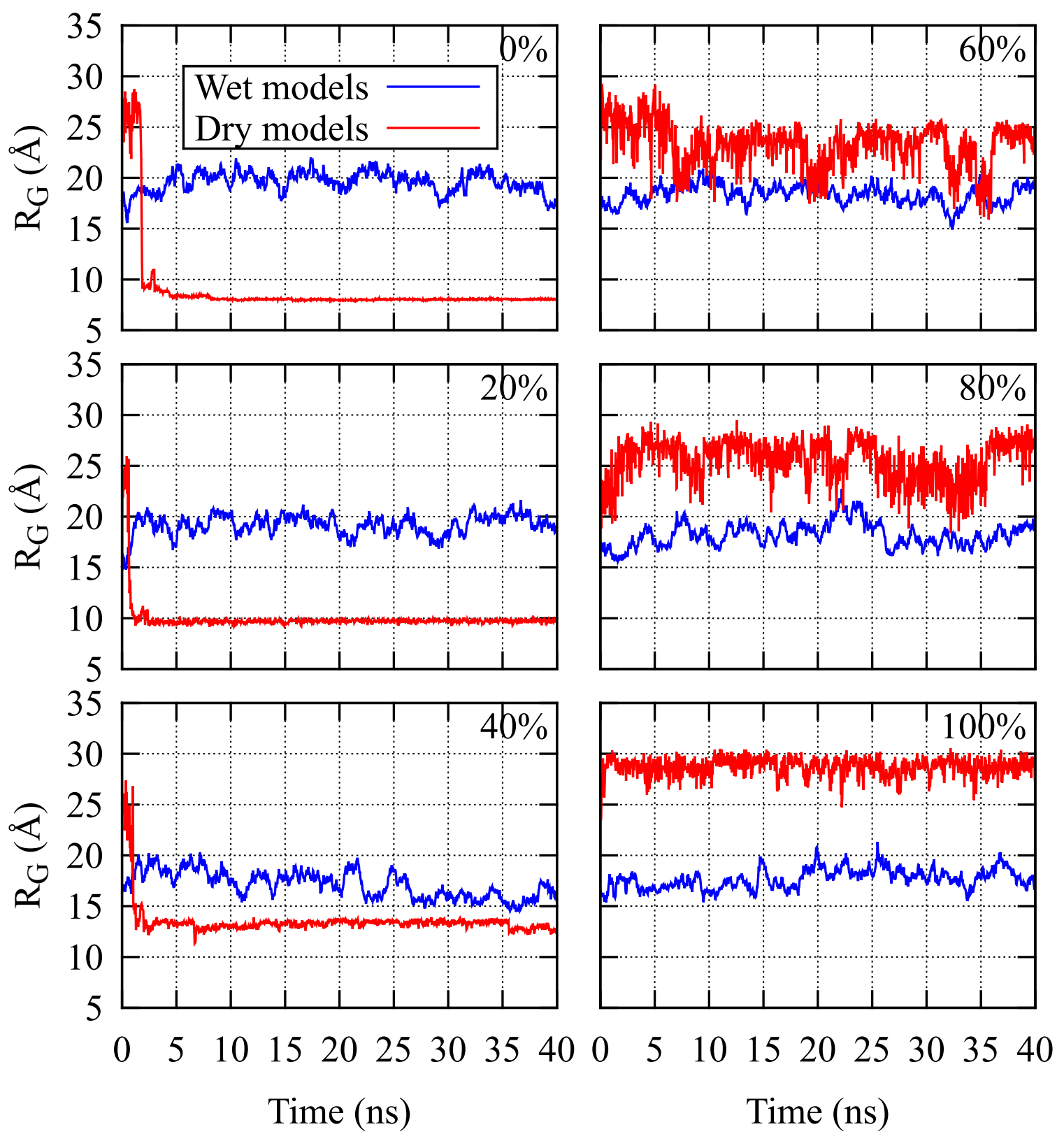

Figure S2: Time evolution of $R_{\mathrm{G}}$ for the set of protonated models. Averages taken at intervals of $20 \mathrm{ps}$. Both the equilibration (0-20 ns) and production runs (20-40 ns) are shown. 

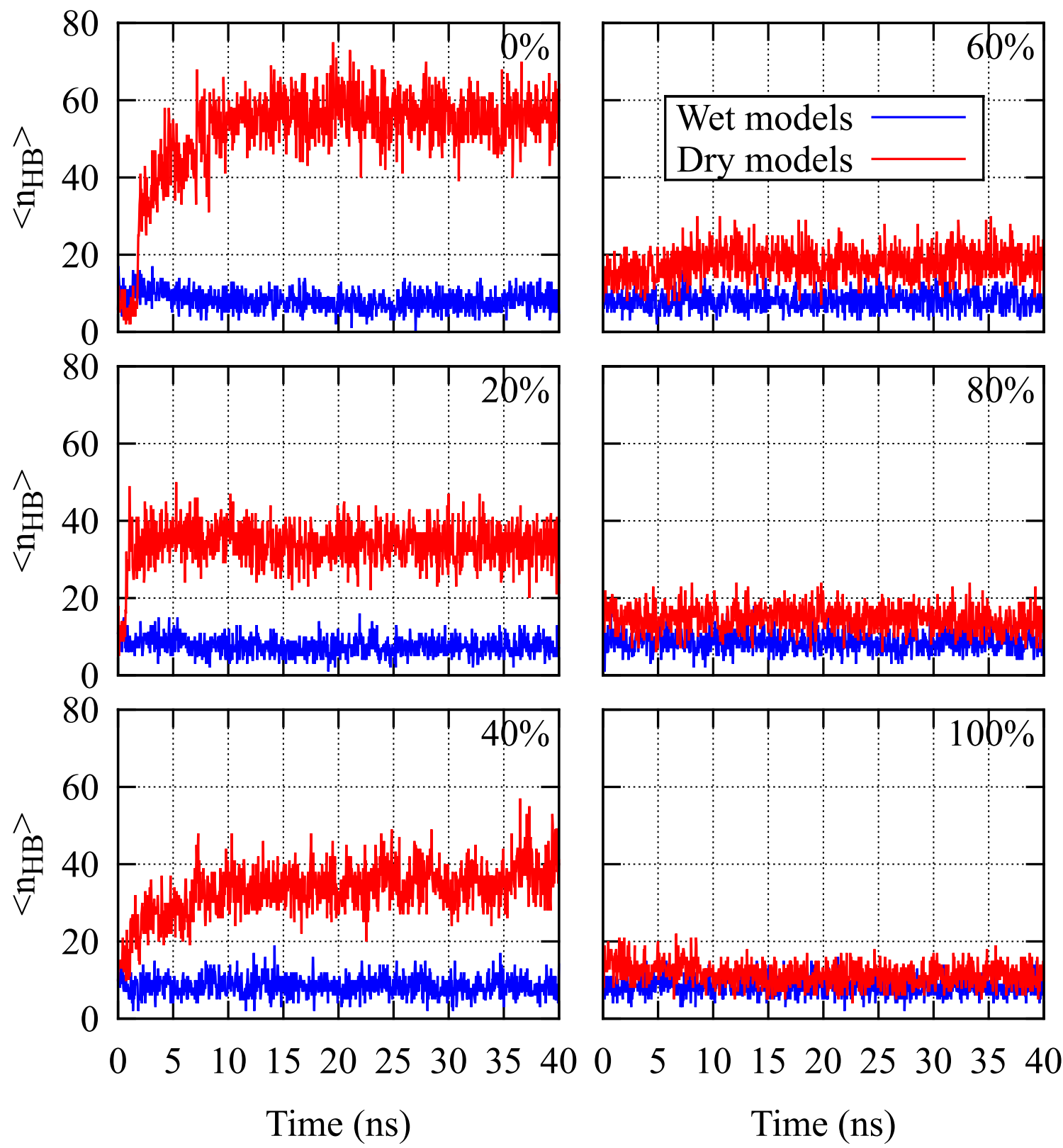

Figure S3: Time evolution of $n_{\mathrm{HB}}$ for the set of protonated models. Averages taken at intervals of $20 \mathrm{ps}$. Both the equilibration (0-20 ns) and production runs (20-40 ns) are shown. 


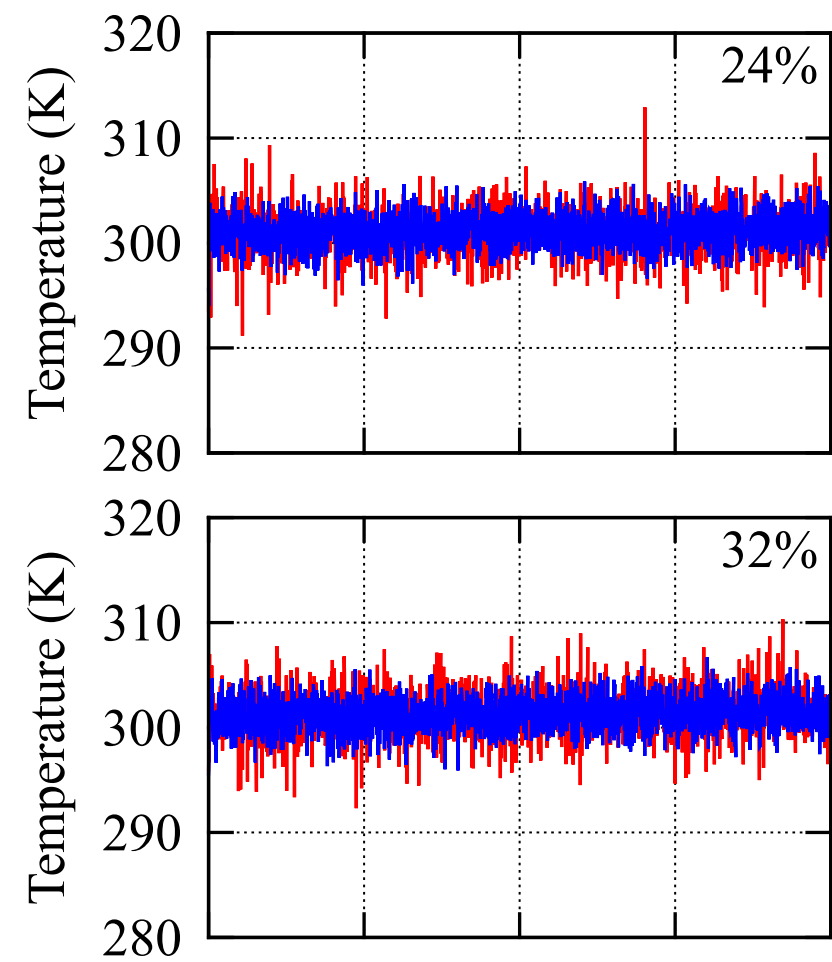

Dry models

Wet models
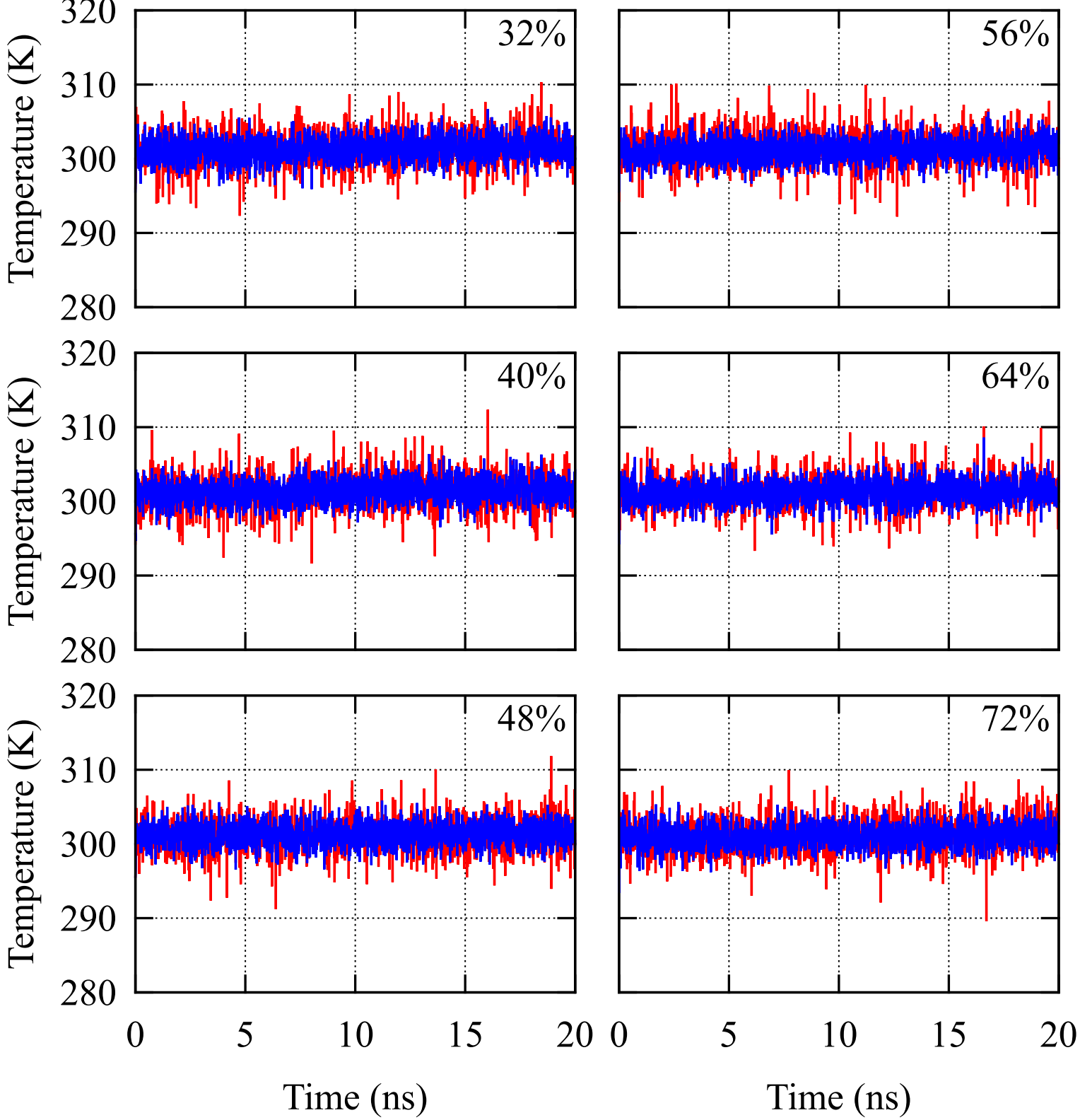

Figure S4: Time evolution of the temperature for the set of crosslinked models. Averages taken at intervals of $10 \mathrm{ps}$. Both the equilibration (0-10 $n s)$ and production runs (10-20 $n s)$ are shown. 

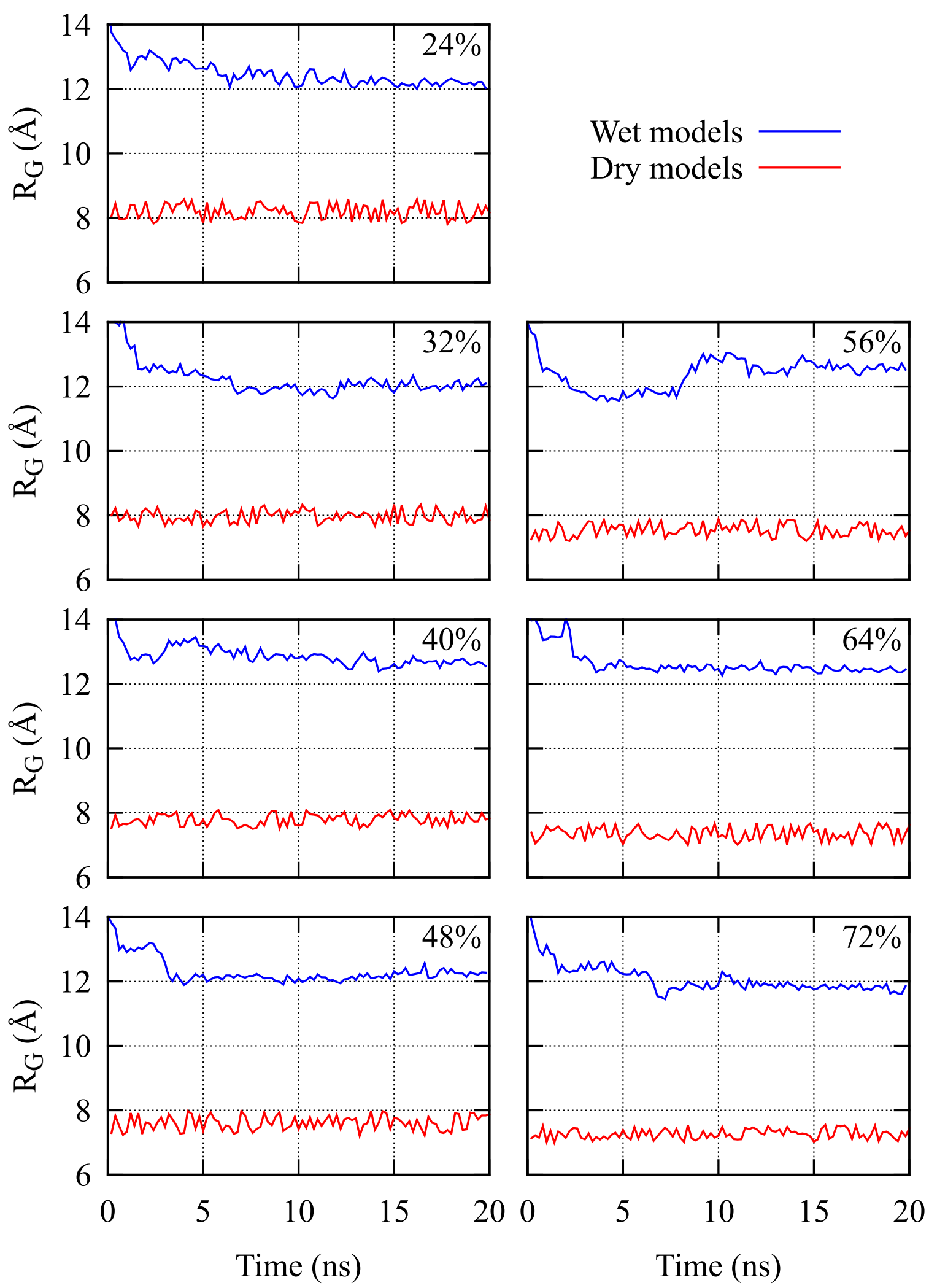

Figure S5: Time evolution of $R_{\mathrm{G}}$ for the set of crosslinked models. Averages taken at intervals of $200 \mathrm{ps}$. Both the equilibration (0-10 $n s)$ and production runs (10-20 ns) are shown. 

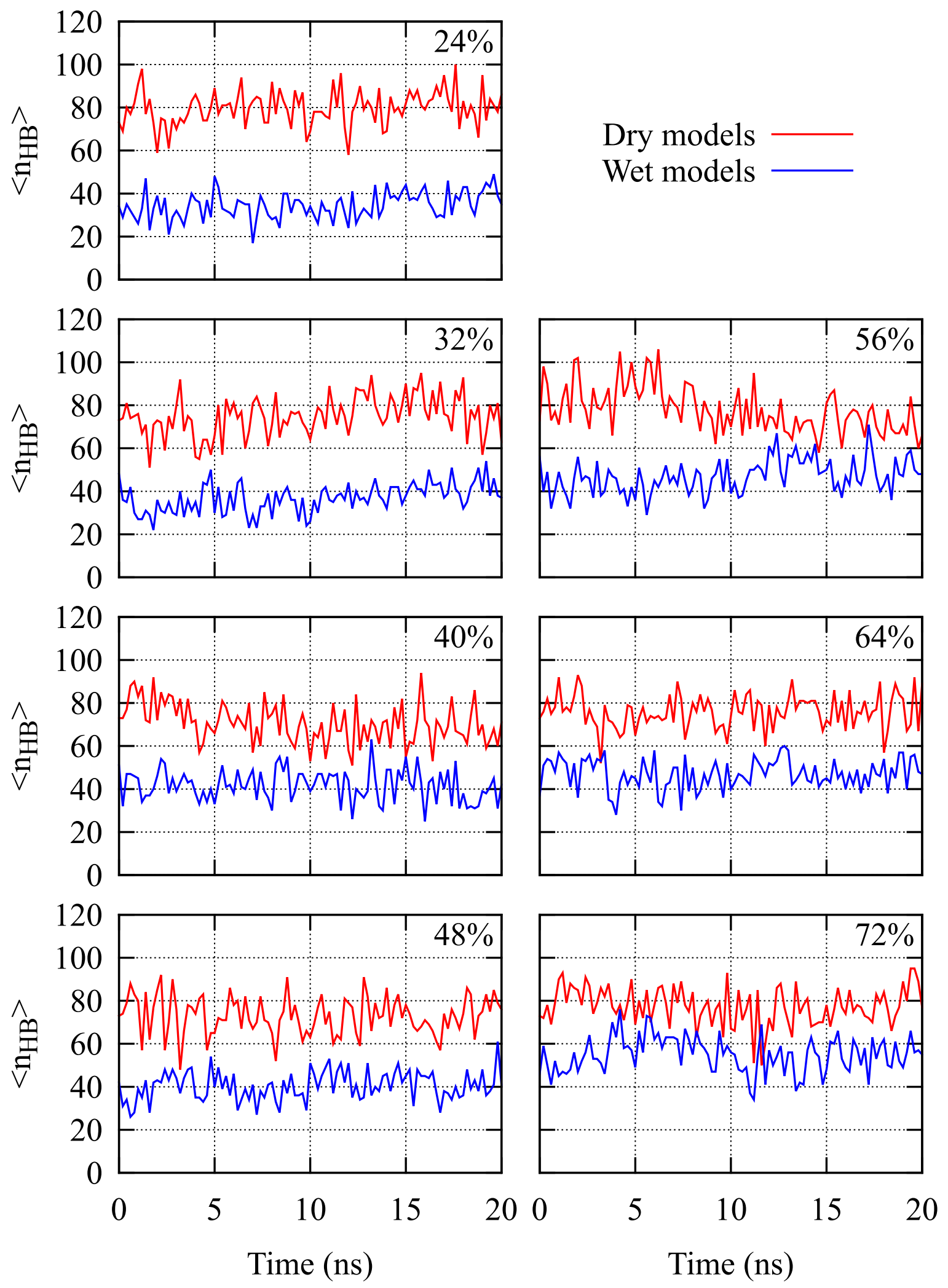

Figure S6: Time evolution of $n_{\mathrm{HB}}$ for the set of crosslinked models. Averages taken at intervals of $200 \mathrm{ps}$. Both the equilibration (0-10 $n s)$ and production runs (10-20 $n s)$ are shown. 